\title{
Generic phase diagram of active polar films
}

\author{
R.Voituriez, ${ }^{1}$ J.F. Joanny, ${ }^{1}$ and J. Prost ${ }^{1,2}$ \\ ${ }^{1}$ Physicochimie Curie (CNRS-UMR168), Institut Curie, \\ Section de Recherche, 26 rue d'Ulm 75248 Paris Cedex 05 France \\ 2 E.S.P.C.I, 10 rue Vauquelin, 75231 Paris Cedex 05, France
}

(Dated: October 30, 2018)

\begin{abstract}
We study theoretically the phase diagram of compressible active polar gels such as the actin network of eukaryotic cells. Using generalized hydrodynamics equations, we perform a linear stability analysis of the uniform states in the case of an infinite bidimensional active gel to obtain the dynamic phase diagram of active polar films. We predict in particular modulated flowing phases, and a macroscopic phase separation at high activity. This qualitatively accounts for experimental observations of various active systems, such as acto-myosin gels, microtubules and kinesins in vitro solutions, or swimming bacterial colonies.
\end{abstract}

PACS numbers: 87.10.+e, 83.80.Lz, 61.25.Hq

Active materials are a challenging class of systems driven out of equilibrium by an internal or an external energy source. Examples of active systems are selfpropelled particle assemblies in bacterial colonies [1, 2], or the membrane and the cytoskeleton of eukaryotic cells [3]. The cell cytoskeleton is a network of long filaments made by protein assembly, interacting with other proteins [4] which can, among other things, crosslink or cap the filaments. Motor proteins, myosins, kinesins or dyneins use the chemical energy of Adenosinetriphosphate (ATP) hydrolysis to "walk" along the filaments, and exert stresses that deform the network [5], leading to an active behavior. The active character of the cytoskeleton plays a major role in most cell functions such as intracellular transport, motility and cell division.

The cell cytoskeleton has a rich and complex dynamical behavior [5, 6, 7, 8, 9]. Self-organized patterns, such as asters, vortices, and rotating spirals, microscopic and macroscopic phase separations ("superprecipitation" [10] ) have been observed as a function of motor and ATP concentrations in a thin film [5]. This two-dimensional geometry gives for example a good description of the thin lamellipodium of a cell spreading or moving on a substrate. Some of these structures have been recently explained theoretically [11, 12], but a full phase diagram of active polar films is still missing.

The passive visco-elastic properties of the cytoskeleton are similar to that of a physical gel made of the cross-linked semi-flexible filaments. Recently, Kruse et al. 12] have proposed a generalized hydrodynamic theory to describe macroscopically the active character of incompressible polar gels, based on conservation laws and symmetry considerations. In this letter, we use the generic model of Ref.[12] to study the stability of compressible active polar films. We perform a linear stability analysis of the uniform states in the case of an infinite two-dimensional geometry, and obtain the dynamic phase diagram. Our results qualitatively account for the experimental observations on various active systems, such as acto-myosin gels, microtubules and kinesin solutions in vitro or swimming bacterial colonies. We choose the example of infinite acto-myosin films that we consider as two-dimensional, and non interacting with the environment. Such an example could be realized by a freely suspended film such as those studied for liquid crystals. We consider the long wavelength limit, and all variables are implicitly averaged over the film thickness. The actin network has a local macroscopic polarization given by a unitary vector $\mathbf{p}=(\cos \theta, \sin \theta)$. This describes degenerate parallel boundary conditions at the film interface, but also equivalently normal boundary conditions with a splayed state. Since the film thickness can vary, the twodimensional actin network is compressible, even though the three-dimensional material is not. The gel has a weakly fluctuating density $c(\mathbf{r})=c_{0}+\rho(\mathbf{r})$. The average density $c_{0}$ can be set to 1 by rescaling the various coefficients of the free energy given in Eq1 below. As a first approximation we neglect the interactions with the solvent and use a one fluid model.

The free energy of the gel, up to quadratic order, couples the polarization $\mathbf{p}$ to the density fluctuation $\rho$ :

$$
\begin{aligned}
F= & \int d x d y\left[\frac{K_{1}}{2}(\nabla \cdot \mathbf{p})^{2}+\frac{K_{3}}{2}(\nabla \times \mathbf{p})^{2}+k \nabla \mathbf{p}-\frac{1}{2} h_{\|} \mathbf{p}^{2}\right. \\
& \left.+w \rho \nabla \cdot \mathbf{p}+\frac{\beta}{2}(\nabla \rho)^{2}+\frac{\alpha}{2} \rho^{2}\right]
\end{aligned}
$$

The splay and bend elastic moduli are assumed to be equal $\left(K_{1}=K_{3}=K\right)$ for the sake of simplicity. The Lagrange multiplier $h_{\|}$enforces the constraint $\mathbf{p}^{2}=1$. The spontaneous splay term leads to boundary terms that are irrelevant in the infinite system size limit [13]. The variation of the free energy with density is characterized by the positive compressibility $\alpha$ and by the positive coefficient $\beta$ associated to the density fluctuation correlation length; $w$ is a coupling constant between density fluctuations and splay. The molecular field, conjugate to the polarization is $h_{\alpha}=-\delta F / \delta p_{\alpha}$ with coordinates $\left(h_{\|}, h_{\perp}\right)$ 
parallel and perpendicular to the polarization. The free energy is similar to that of a ferro-electric nematic liquid crystal when the order parameter $\mathbf{n}$ does not have a fixed length [14, 15]).

The gel motion is described by the two-dimensional velocity field $\mathbf{v}$. The strain rate tensor is $u_{\alpha \beta}=\left(\partial_{\alpha} v_{\beta}+\right.$ $\left.\partial_{\beta} v_{\alpha}\right) / 2$, and the vorticity tensor $\omega_{\alpha \beta}=\left(\partial_{\alpha} v_{\beta}-\partial_{\beta} v_{\alpha}\right) / 2$. The conservation equation of the gel is written as: $\partial_{t} \rho+$ $\partial_{\alpha}(1+\rho) v_{\alpha}=0$.

The gel is driven out of equilibrium by continuous and homogeneous input of energy, characterized by the chemical potential difference $\Delta \mu$ between ATP and its hydrolysis products, which we assume to be constant.

The gel dynamics is described by the linear hydrodynamic equations for active polar gels of Ref.[12]. The constitutive equations for the mechanical deviatory stress tensor $\sigma_{\alpha \beta}$ and the rate of change of the polarization $\frac{D}{D t} p_{\alpha}=\frac{\partial p_{\alpha}}{\partial t}+\left(v_{\gamma} \partial_{\gamma}\right) p_{\alpha}+\omega_{\alpha \beta} p_{\beta}$ read, at long time scales when the gel behaves as a viscous liquid:

$$
\begin{aligned}
2 \eta u_{\alpha \beta}= & \sigma_{\alpha \beta}+\zeta \Delta \mu p_{\alpha} p_{\beta}+\bar{\zeta} \Delta \mu \delta_{\alpha \beta}-\frac{\nu}{2}\left(p_{\alpha} h_{\beta}+p_{\beta} h_{\alpha}\right) \\
& -\bar{\nu} p_{\gamma} h_{\gamma} \delta_{\alpha \beta}+\frac{1}{2}\left(p_{\alpha} h_{\beta}-p_{\beta} h_{\alpha}\right) \\
\frac{D p_{\alpha}}{D t}= & \frac{1}{\gamma} h_{\alpha}+\lambda p_{\alpha} \Delta \mu-\nu u_{\alpha \beta} p_{\beta}-\bar{\nu} u_{\beta \beta} p_{\alpha}
\end{aligned}
$$

We neglect here the geometric non-linearities introduced in [12]. The rotational viscosity $\gamma$ and the coupling constants between flow and polarization $\nu, \bar{\nu}$ are standard liquid crystal parameters [16]. The active contributions to the mechanical stress and to the rate of change of the polarization are proportional to $\Delta \mu$ and are characterized by the coefficients $\zeta, \bar{\zeta}$ and $\lambda$. This set of constitutive equations is completed at low Reynolds numbers by the force balance: $\partial_{\alpha}\left(\sigma_{\alpha \beta}-\Pi \delta_{\alpha \beta}\right)=0$. Locally, there are two forces acting on the gel, the deviatory stress tensor $\sigma_{\alpha \beta}$ and the pressure $\Pi=\frac{\delta F}{\delta \rho}=w \nabla \cdot \mathbf{p}+\alpha \rho-\beta \Delta \rho$.

It is observed experimentally [17], and predicted by 1dimensional models [6] that the overall effect of myosin II motors on actin solutions is contractile. This corresponds to negative values of both $\zeta$ and $\bar{\zeta}$. The molecular motors also have an effect on the rate of change of the polarization described by $\lambda$; if $\lambda>0$ the polarization is enhanced. This seems to be observed experimentally (zipping effect in Ref.[18]). We consider here only ordered polar phases with a unitary polarization vector $\mathbf{p}$, and we ignore this active coupling (setting $\lambda=0$ ) for simplicity [19].

In order to discuss the accessible steady states of an infinite active gel film, we first study the states of uniform polarization and velocity gradient. These asymptotic states are non-equilibrium states, and cannot therefore be obtained by minimizing a free energy functional. In a two-dimensional geometry, the hydrodynamic equations of motion give 11 scalar equations for the 11 independent variables $\mathbf{x} \equiv \theta, \rho, h_{\alpha}, v_{\alpha}, \sigma_{\alpha \beta}, \pi$. These equations of motion have two types of homogeneous steady states: a static state where the velocity gradient $u_{\alpha \beta}$ vanishes and the polarization is uniform and oriented in a direction $\theta_{0}$; and a flowing state with a finite velocity gradient and a uniform polarization. In two dimensions, the most general steady flow with a constant velocity gradient is a superposition of two simple shear flows in two perpendicular directions $x$ and $y$. The only non vanishing components of the velocity gradient are $\partial_{x} v_{y}$ and $\partial_{y} v_{x}$. We consider here for simplicity only one component shear flows for which $\partial_{y} v_{x}=0$. The velocity is then along the $y$ direction and the polarization angle $\theta_{\nu}$ is such that $\cos \left(2 \theta_{\nu}\right)=1 / \nu$ (we assume $|\nu|>1$ ). This flowing state is the analog, for an infinite compressible gel, of the flowing state obtained in a confined geometry in [13] and to the rotating spirals in a cylindrical geometry described in [12]. It confirms the possibility of obtaining spontaneous flows in polar active materials.

The stability of the two homogeneous states is studied by introducing a small perturbation at point $\mathbf{r}$, at time $t$ with a wavevector $\mathbf{k}$ and a growth rate $s: \mathbf{x}=\mathbf{x}_{0}+$ $\mathbf{x}_{1} \exp [s t+i \mathbf{k r}]$, where $\mathbf{x}_{0}$ is a steady state solution. The equation for the perturbation $\mathbf{x}_{\mathbf{1}}$ can formally be written in matrix form $M \mathbf{x}_{\mathbf{1}}=\mathbf{0}$ and the possible growth rates of the perturbation are determined from the equation $\operatorname{det}(M)=0$. This is a quadratic equation in $s$ with two roots denoted by $s^{+}, s^{-}$with $\operatorname{Re}\left(s^{+}\right) \geq \operatorname{Re}\left(s^{-}\right)$. The sign of $\operatorname{Re}\left(s^{+}\right)$gives the stability limit of the steady state homogeneous phase.

We are only able to give a complete discussion of the stability of the uniform steady states with respect to a periodic perturbation in the quasi 1-dimensional case where we do not allow for a $y$ dependence $\left(k_{y}=0\right)$. The treatment of the fully general 2-dimensional problem requires numerical work. However most of the physics can be extracted from the quasi-1-dimensional case, which we present hereafter; the numerical study allows us to extend our conclusions to the general 2-dimensional case.

We first analyze the stability of the static state, fixing all the parameters of the active gel introduced above, except for the Franck constant $K$ and the active stress $\zeta \Delta \mu$. As there is no flow in the system, there is one single direction, given by the polarization, which we choose as the $y$ axis $\left(\theta_{0}=\pi / 2\right)$. The numerical study of the 2-dimensional problem reveals that the maximum of $\operatorname{Re}\left[s^{+}\left(k_{x}, k_{y}\right)\right]$ lies on the axis $k_{y}=0$, for any value $\zeta \Delta \mu \leq \zeta \Delta \mu^{*}(K)$, with $\zeta \Delta \mu^{*}(K)>0$. In this regime, it is therefore sufficient to consider one-dimensional perturbations in the $x$ direction. The growth rates are then solutions of: 
$2 \eta \gamma a s^{2}+s\left[(2 \eta \beta+K a b \gamma) k^{2}+2 \eta \alpha+\zeta \Delta \mu \gamma a(\nu+1)\right]+b K \beta k^{4}+\left[b\left(K \alpha-w^{2}\right)+\zeta \Delta \mu \beta(\nu+1)\right] k^{2}+\zeta \Delta \mu \alpha(\nu+1)=0$

where we have redefined $k_{x} \equiv k$ and where $a$ and $b$ are dimensionless functions of the parameters: $a=$ $2 \frac{\eta}{\gamma}+\bar{\nu}(\nu+\bar{\nu})$ and $b=2 \frac{\eta}{\gamma}+\frac{(1+\nu)^{2}}{2}$. It is useful to analyze first the passive case where $\zeta \Delta \mu=0$. If $K \geq w^{2} / \alpha$, $\operatorname{Re}\left(s^{+}\right)$is maximum and negative for $k=0$ and the active gel is stable. If $K<w^{2} / \alpha$, one can check that $\operatorname{Re}\left(s^{+}\right)$ is maximum and positive when $k=k_{c} \neq 0$. The uniformly ordered phase is locally unstable with respect to a finite wavelength longitudinal mode transverse to the ordering direction. It follows that in this region of the phase diagram there necessarily exists an ordered modulated phase characterized by a periodic polarization. The precise symmetry of this passive phase, (striped, hexagonal or cubic phase), has been discussed in [14, 15] for the case of ferroelectric nematic liquid crystals. We now consider the effect of the activity on this instability. For $\zeta \Delta \mu<0$, the static state is unstable at zero wave vector (both growth rates are real and $s^{+} s^{-}<0$ ), with respect to the flowing state, as found in Ref.[13]. For $\zeta \Delta \mu>0$, the uniform state is unstable with respect to a finite wavelength longitudinal mode, transverse to the polarization direction when the Franck constant is small enough $K<K_{c}(\zeta \Delta \mu)$. The function $K_{c}(\zeta \Delta \mu)$ can be analytically calculated from Eq⿴囗十 in this regime and is plotted in Fig 1. For $\zeta \Delta \mu>\zeta \Delta \mu^{*}(K)>0$, a 2 -dimensional numerical analysis is required and reveals that for $K<K_{c}(\zeta \Delta \mu)$ (evaluated by numerically computing the sign of $\operatorname{Re}\left(s^{+}\right)$), there are 2 independent most unstable wavevectors $\mathbf{k}_{c 1}$, and $\mathbf{k}_{c 2}$.

We now discuss the flowing state $(\zeta \Delta \mu<0)$, which we believe to be relevant for cytoskeleton dynamics. (If $\zeta \Delta \mu>0$, the flowing state is unstable at any wave vector). The numerical study of a 2-dimensional perturbation shows that the maximum of the growth rate $\operatorname{Re}\left[s^{+}\left(k_{x}, k_{y}\right)\right]$ is obtained for a wavevector $\mathbf{k}$ perpendicular to the polarization, for any value $\zeta \Delta \mu \leq 0$. It is therefore sufficient to consider an effective 1-dimensional problem where spatial variations are allowed only along the unstable direction orthogonal to $\mathbf{p}$. The growth rates are in this case solutions of :

$$
2 \eta \gamma a s^{2}+s\left[(2 \eta \beta+K a b \gamma) k^{2}+2 \eta \alpha^{\prime}\right]+b K \beta k^{4}+b\left(K \alpha-w^{2}\right) k^{2}+i k d w \zeta \Delta \mu-\zeta \Delta \mu \alpha(\nu+1)=0
$$

where $\alpha^{\prime}$ is an effective compressibility, and $d$ a dimensionless function of the parameters: $d=2 \frac{\eta}{\gamma}+(\nu+1) / 2+\bar{\nu}$ and $\alpha^{\prime}=\alpha+d \gamma \zeta \Delta \mu(\nu+1) / 2$.

For $K<K_{c}(\zeta \Delta \mu)$ (evaluated by numerically computing the sign of $\operatorname{Re}\left(s^{+}\right)$from Eq(5), the uniform state is unstable with respect to a finite wavelength mode. The value of the critical Franck constant $K_{c}$ diverges for an active stress $\zeta \Delta \mu^{c}$ such that $\alpha^{\prime}\left(\zeta \Delta \mu^{c}\right)=0$. At lower values of the active stress, the flowing state is always unstable. The most unstable wave vector $\left|\mathbf{k}_{c}\right|$ decreases with $\zeta \Delta \mu$ and vanishes if the active stress is smaller than a critical value $\zeta \Delta \mu_{p}(K)$. Note that in this regime of negative active stress, $\operatorname{Im}\left(s^{+}\right) \neq 0$ and the instability is oscillatory, as opposed to the regime $\zeta \Delta \mu>0$.

The results of the stability analysis are summarized in the phase diagram of Fig,1. A full analysis of the nonlinearities is necessary to predict the symmetry of the dynamic equilibrium states. This would require an exhaustive study of all the possible quadratic and cubic terms in the perturbations in the equations of motion, which seems out of reach. We can nevertheless infer qualitatively the structure of the different phases.
In regions $\mathrm{B}^{-}$and $\mathrm{B}^{+}$of the phase diagram, the instability occurs at finite wavevector. It is similar to the instability of the passive system described in [14, 15]: it favors splay in the system, which can appear either in a striped or "lattice" (cubic, hexagonal, or triangular) phase. Because of the outbreak of polarization gradients, the strain rate tensor cannot vanish and the system flows. The continuity of the velocity field imposes extra constraints and does not allow for the formation of domain walls. This, together with the results of [12] suggests that in the lattice phases, each elementary cell contains a spiral-like structure with a rotating flow. This structure is not compatible with the hexagonal symmetry : the only possible lattice phases have therefore cubic or triangular symmetries sketched on Fig 1 .

In region $\mathrm{C}^{-}$, the system is unstable for $\mathbf{k}=0$ and thus its behavior depends on boundary conditions. We expect a macroscopic phase separation since the effective compressibility $\alpha^{\prime}$ becomes negative. In region $\mathrm{C}^{+}$, the existence of 2 independent unstable modes suggests that an oblique phase prevails, even if non linear terms could a priori select only one of the unstable modes. 


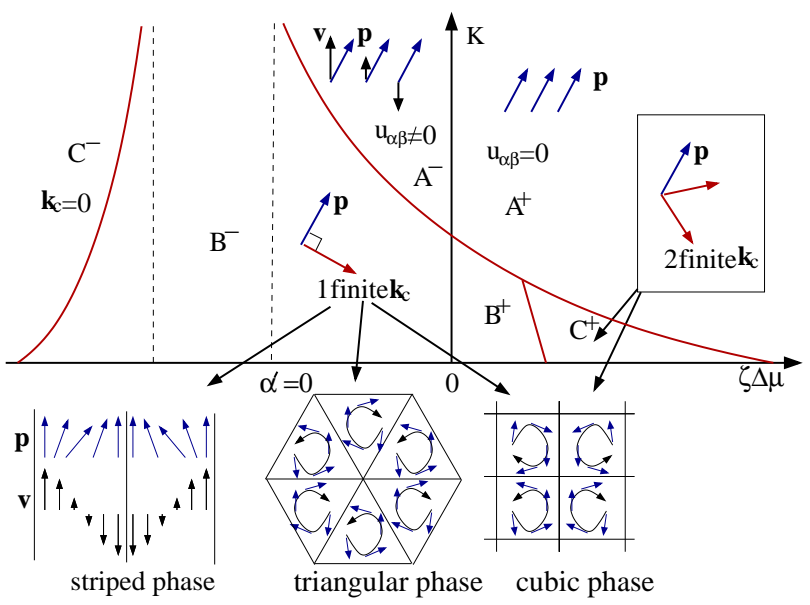

FIG. 1: Dynamic phase diagram of an active polar film. The transition line between regions $B^{+}$and $C^{+}$is the line $\zeta \Delta \mu^{*}(K)$; the transition between regions $B^{-}, B^{+}$and $C^{+}$ and regions $A^{-}$and $A^{+}$is the line $K_{c}(\zeta \Delta \mu)$; the transition between regions $B^{-}$and $C^{-}$is the line $\Delta \mu_{p}(K)$

Our analysis has been presented in terms of the actin cytoskeleton, but it is general enough to be applied to any visco-elastic, polar, and active material. We now briefly discuss two other examples. One should keep in mind that our model does not include noise, be it thermal or intrinsic (due to the stochastic activity of motors, actin polymerization...) and that the predicted ordered phases could be disordered in the presence of noise.

Systematic quantitative data describing the phases of in vitro acto-myosin solutions as a function of the contractility (associated to changes either in myosin II and/or ATP concentrations) and therefore of $\zeta \Delta \mu$ do not seem to be available yet. However, both disordered vortex flowing phases [11, 17], which could correspond to region $\mathrm{B}^{-}$, and phase separations (superprecipitation, 10], corresponding to region $\mathrm{C}^{-}$) are observed in experiments or obtained by numerical simulations. Microtubules and kinesins solutions also belong to the class of materials described by our model. They are viscous, polar, and active. The phase diagram obtained in this paper qualitatively agrees with the phases observed in vitro for microtubules and kinesin motors in two dimensions [5]: when the motor concentration is increased, a flowing phase of spirals appears (B); for higher motor concentrations, the spirals become growing asters which progressively separate $\left(\mathrm{C}^{-}\right)$; for even higher concentrations microtubules bundles are formed $\left(\mathrm{C}^{-}\right)$.

A similar behavior is observed in bacterial colonies : experiments on 2-dimensional colonies of B. Subtilis [2] show a bacterial flow composed of rotating whirls of swimming bacteria. This bacterial turbulence could be described by a disordered version of the cubic phase $\left(\mathrm{B}^{+}\right.$, $\mathrm{C}^{+}$or $\mathrm{B}^{-}$) predicted by our model. Indeed these bacteria are rod-like shaped, oriented by their flagella, and therefore polar; in a coarse-grained picture, a colony can be viewed as a viscoelastic gel; last, they consume chemical energy $\left(0_{2}\right)$ and hence are active. The alternative model of [1] also predicts an intrinsic flow instability for self propelled particle assemblies. This instability differs from the one discussed here in that it has no threshold. The main differences with our description are first that our model in this one fluid version cannot impose a spontaneous velocity of the gel with respect to the background fluid; second, our model describes a compressible gel, this feature being crucial to trigger the instabilities. A thorough comparison between the two theories needs a two fluids description and is underway.

We are grateful to F. Amblard (Institut Curie), D. R. Nelson (Harvard University), D. Riveline (Grenoble) and S. Ramaswamy (Bangalore) for very useful discussions.

[1] R.A. Simha and S. Ramaswamy, Phys. Rev. Lett. 89, 058101 (2002); Y. Hatwalne, S. Ramaswamy, M. Rao and R.A. Simha, Phys. Rev. Lett. 92, 118101 (2004)

[2] C. Dombrowski, L. Cisneros, S. Chatkaew, R. E. Goldstein, and J. O. Kessler Phys. Rev. Lett. 93, 098103 (2004)

[3] B. Alberts et al., Molecular Biology of the Cell 4th ed. (Garland, New York, 2002).

[4] J. Howard, Mechanics of Motor Proteins and the Cytoskeleton (Sinauer Associates, Inc., Sunderland, 2001).

[5] F.J. Nédélec et al., Nature 389, 305 (1997).

[6] K. Kruse and F. Jülicher, Phys. Rev. Lett. 85, 1778 (2000); K. Kruse, S. Camalet, and F. Jülicher, Phys. Rev. Lett. 87, 138101 (2001).

[7] H.Y. Lee and M. Kardar, Phys. Rev. E 64, 056113 (2001).

[8] J. Kim et al., J. Korean Phys. Soc. 42, 162 (2003).

[9] T.B. Liverpool and M.C. Marchetti, Phys. Rev. Lett. 90, 138102 (2003).

[10] T. Sekine and M. Yamaguchi, J. Biochem 59(2), 195 (1966); Y. Nonomura and S.J. Ebashi, Mechanochem. Cell Motil. 3(1), 1 (1974)

[11] S. Sankararaman, G.I. Menon and P.B. Sunil Kumar, Phys. Rev. E 70, 031905 (2004), M.C. Aronson Unpublished

[12] K. Kruse, J.F. Joanny, F. Jülicher, J. Prost and K. Sekimoto, Phys. Rev. Lett 92, 078101 (2004) and Eur. Phys. J. E 16, 5 (2005).

[13] R. Voituriez, J.F. Joanny and J. Prost, Eur. Phys. Lett. 70, 3 (2005).

[14] G.A. Hinshaw Jr., R.G. Petschek and R.A. Pelcovits, Phys. Rev. Lett. 60, 18 (1988).

[15] D. Blankschtein and R.M. Hornreich Phys. Rev. B 32, 3214 (1985).

[16] P.G. De Gennes and J. Prost, The Physics of Liquid Crystals (Clarendon Press, Oxford 1993).

[17] K. Takiguchi, J. Biochem 109, 502 (1991)

[18] J. Uhde, M. Keller, E. Sackmann, A. Parmeggiani, and E. Frey Phys. Rev. Lett. 93, 268101 (2004)

[19] We also considered the case where $\zeta$ and $\bar{\zeta}$ vanish and $\lambda$ is varied. There is no phase separation in this case. 